\title{
B waves: a systematic review of terminology, characteristics, and analysis methods
}

\author{
Isabel Martinez-Tejada ${ }^{1,2^{*}} \mathbb{0}$, Alexander Arum ${ }^{1}$, Jens E. Wilhjelm², Marianne Juhler ${ }^{1}$ and Morten Andresen ${ }^{1}$
}

\begin{abstract}
Background: Although B waves were introduced as a concept in the analysis of intracranial pressure (ICP) recordings nearly 60 years ago, there is still a lack consensus on precise definitions, terminology, amplitude, frequency or origin. Several competing terms exist, addressing either their probable physiological origin or their physical characteristics. To better understand B wave characteristics and ease their detection, a literature review was carried out.

Methods: A systematic review protocol including search strategy and eligibility criteria was prepared in advance. A literature search was carried out using PubMed/MEDLINE, with the following search terms: B waves + review filter, slow waves + review filter, ICP B waves, slow ICP waves, slow vasogenic waves, Lundberg B waves, MOCAIP.

Results: In total, 19 different terms were found, $B$ waves being the most common. These terminologies appear to be interchangeable and seem to be used indiscriminately, with some papers using more than five different terms. Definitions and etiologies are still unclear, which makes systematic and standardized detection difficult.

Conclusions: Two future lines of action are available for automating macro-pattern identification in ICP signals: achieving strict agreement on morphological characteristics of "traditional" B waveforms, or starting a new with a fresh computerized approach for recognition of new clinically relevant patterns.
\end{abstract}

Keywords: Intracranial pressure, B waves, Slow waves, Vasogenic waves

\section{Background}

Intracranial pressure (ICP) monitoring plays an important role in the management of patients with many neurological and neurosurgical disorders. In the 1960s, Lundberg described typical macro-patterns: A, B and C waves [1]. $\mathrm{B}$ waves were defined as short repeating elevations in ICP $(10-20 \mathrm{mmHg})$ with a frequency of $0.5-2$ waves $/ \mathrm{min}$. These classic B wave patterns may be seen in ICP monitoring in intensive care unit settings (ICU), but ICP is also monitored in a large number of brain diseases covering a spectrum from acute and subacute ICU settings to elective outpatient follow-up. Today a large proportion of patients undergo ICP monitoring for milder degrees of disease where pathological patterns are not as prominent. In such scenarios, wave patterns are still called B waves but differ in amplitude and

\footnotetext{
*Correspondence: imate@dtu.dk

${ }^{1}$ Clinic of Neurosurgery, Copenhagen University Hospital, Rigshospitalet, Copenhagen, Denmark

Full list of author information is available at the end of the article
}

visual appearance from those defined by Lundberg. Such 'uncharacteristic' B waves are often smaller in amplitude and appear as an irregular pattern, but they have not yet been formally classified. The current paper uses B waves as an encompassing umbrella for all variations.

The source of B waves is unknown and although they are mostly associated with cerebral dysfunction, their clinical significance is unclear, as they may also appear as normal physiological phenomena [2,3]. Their source is most commonly related to vasogenic activity, but an origin from a neuro-pacemaker system has also been suggested [4]. This diverging information poses a challenge to a consensus for a general description of $\mathrm{B}$ waves and their quantification, hindering their identification during diagnosis and treatment of different diseases categories. Because of these difficulties, clinical practice outside specialized centers with a focus on ICP-related research is currently largely restricted to readings of mean ICP.

Identification of waveform abnormalities by simple visual inspection is still a common clinical practice. This has 
an obvious bias from reliance on personal empiric experience and raises questions of interobserver reproducibility. Automated and standardized detection of B wave patterns would increase the usefulness in both clinical and research settings. This automated detection is only possible if the waveform morphological characteristics are clearly defined; preferably by consensus in the scientific community. A systematic quantitative detection system could allow for identification of B wave variations and other 'non-Lundberg' patterns, replacing traditional visual inspection.

The aim of this study was to assess the various terms and definitions used to describe classical B waves in order to highlight the lack of consensus in terms of terminology and morphological characteristics, frequency and amplitude. Therefore, a systematic review was carried out to summarize the different terminologies and definitions regarding $B$ waves and the methods used for $B$ wave identification.

\section{Methods}

Relevant studies were identified by a single reviewer using the online database PubMed/Medline. The diagram in Fig. 1 gives an overview of the literature search based on the PRISMA systematic review methodology [5]. Studies were selected if they included the key terms slow vasogenic waves, Lundberg $B$ waves, slow ICP waves, ICP B waves, MOCAIP, B waves + review filter, and slow waves + review filter. A total sum of 816 paper abstracts were screened initially for content relevance and 124 papers were included in the search review.

\section{Results}

\section{Terminologies}

A total of 19 terminologies were found to describe B waves in the reviewed papers (Table 1). The most common terms being $B$ waves and (ICP) slow waves (Fig. 2). Nine articles used four or more terms to refer to $B$ waves. The choice of terminology is often related to the ongoing etiology discussion: 22 articles include the word vasogenic thereby implying cerebrovascular changes as the origin of the waves. Raftopoulos [6], Santamarta [7], Yokota [8], and Kasprowicz [9], defined further subgroups in order to clarify the sources underlying the presence of B waves (Table 2).

\section{Characteristics}

B waves were identified based on two major wave parameters: frequency and amplitude. Frequency is the number of waves that fit into a certain time period, usually measured as waves per minute and $27 \%$ of the papers defined a frequency of $0.5-2$ waves $/ \mathrm{min}$, as originally defined by Lundberg [1]. To accommodate $B$ waves of a lower frequency, the term slow was introduced [10]. The term slow waves was then used to define waves with a frequency window of 0.33 to 3 waves/min [11]. Two other papers extended the frequency upper limit to 4 waves/ $\min [12,13]$.

As mentioned, B waves can also be characterized by their amplitude. Lundberg defined a maximum amplitude of $50 \mathrm{mmHg}$ back in the 1960s. Under pathological conditions, this level of elevation is less often seen to such an extent today, and B waves with lower amplitudes are more likely to be present. As an example, lower amplitude $B$ waves are present in cases of normal pressure hydrocephalus, where the occurrence of $B$ waves is not related to high ICP [14].

\section{Sub-classification}

In addition to frequency and amplitude, two other parameters are generally defined for the analysis of $\mathrm{B}$ waves. B waves can also be characterized by their shape and whether a plateau phase is present or not. The shape is considered symmetrical if the duration of ascending and descending phases is the same. If the ascending phase is longer, then the shape is asymmetrical. The use of these parameters gives rise to different subclasses within B waves (Table 2). All subclasses fit into the traditional definition of $B$ waves with an extended frequency spectrum, but mainly differ in their morphological characteristics (Fig. 3).

Besides these four parameters, Raftopolous et al. and Santamarta et al. also use the duration of the ICP wave to characterize $\mathrm{B}$ waves. They distinguish between three morphological subclasses: (1) small symmetrical waves with an amplitude below $10 \mathrm{mmHg}$, (2) great symmetrical waves with an amplitude above $10 \mathrm{mmHg}$, and (3) intermediate waves, with the same frequency as symmetrical waves but an amplitude similar to plateau waves [6, 7].

Kasprowicz et al. describe three subcategories of B waves based on the investigation of their unique shape: (1) symmetrical ICP B waves, (2) asymmetrical ICP B waves, and (3) slow ICP B waves with plateau phase. They show how the different subtypes of B waves are related to changes in the ICP pulse shape, which indicate that each has a unique origin [9]. Similarly, Yokota et al. also suggest the existence of three subgroups but from the analysis of ICP amplitude and occurrence: (1) episodic B-waves, (2) persistent, high pressure B-waves, and (3) continuous, regular B-waves, and that these patterns may better distinguish between different origins of ICP waves [8].

The intermediate waves described by Raftopolous et al. [6] and Santamarta et al. [7] contain amplitudes similar to plateau waves, Kasprowicz et al. [9] describe B waves 


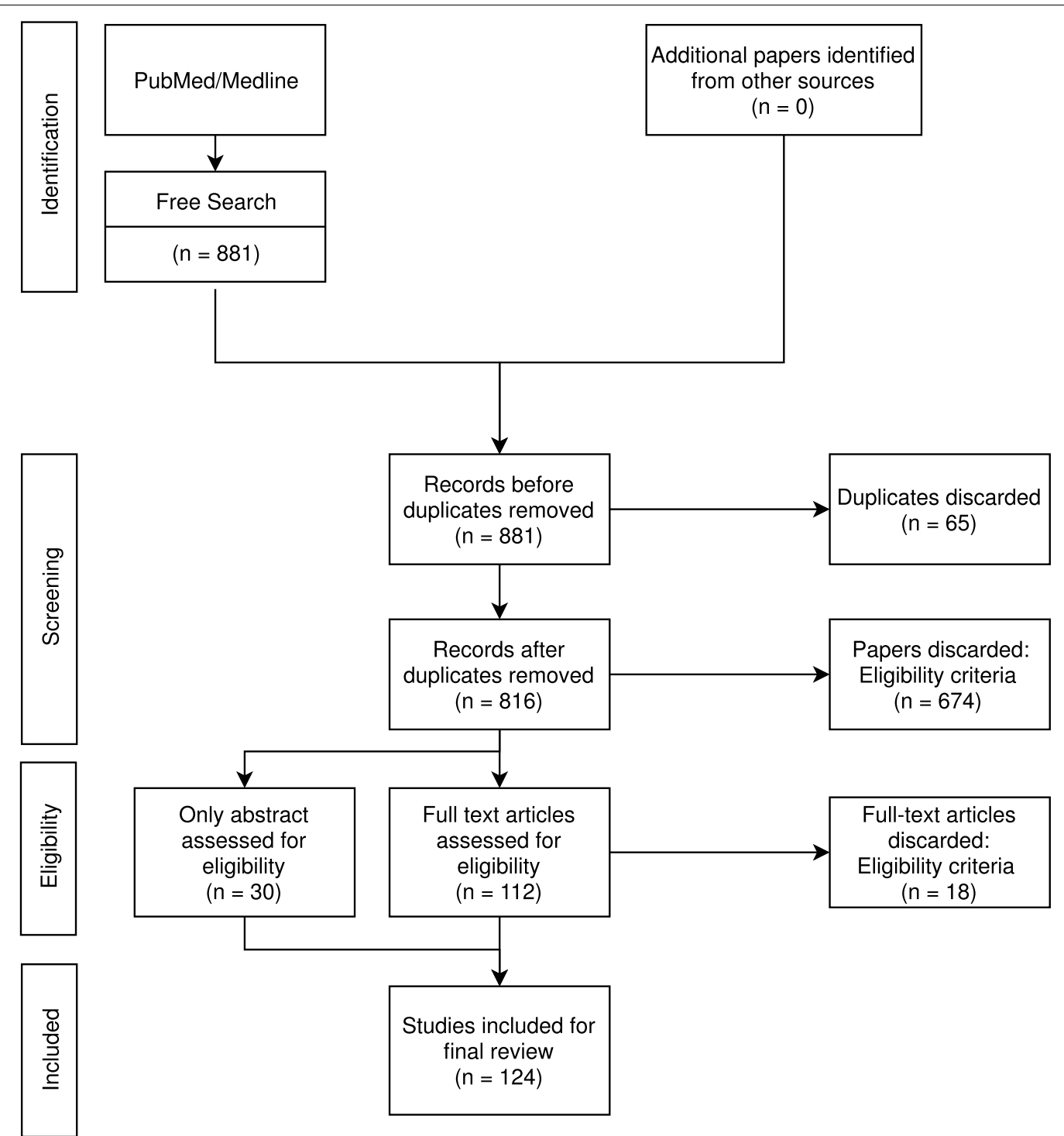

Fig. 1 Modified PRISMA 2009 flow diagram. Systematic literature search and selection process overview. Given that the goal of this literature review was to give an insight into the different terminologies and definitions of B waves, only articles specifically mentioning B waves or related terms were included in the study selection. As an example: slow waves of $A B P$ was not included. Papers simultaneously published by different journals were considered as duplicates and also excluded. The remaining articles $(n=124)$ were thoroughly examined and included in this study following the PRISMA flow-diagram. Terminologies, definitions, and methods were identified individually by two independent reviewers and categorized according to a predefined protocol. Disagreements were resolved by consensus. No importance was given to the order of words, ICPB $B$ waves was treated equally to $B I C P$ waves. Hyphens were removed, $B$-waves were grouped together with $B$ waves. Of and in were disregarded, slow waves of ICP were registered as slow waves ICP. Only terminologies associated with ICP B waves were included (i.e. slow waves of $A B P$ was not included). Terminologies in singular form were registered as plural, $B$ wave was registered as $B$ waves. Terms used less than three times were categorized as other

with a plateau phase, and Yokota et al. [8] describe persistent high pressure $B$ waves. It is noteworthy that all sub-classification attempts contain a B wave subtype with plateau-like features. This raises the question whether there is a continuous transition from B waves to plateau waves or whether they have different etiology.
To summarize, B waves are categorized into different subclasses if they have distinct shapes and/or if their amplitude is different. These sub-classification attempts may be used as supplementary evidence that the classical waveform categories do not adequately address waveforms identified in clinical practice today. 
Martinez-Tejada et al. Fluids Barriers CNS

(2019) 16:33

Page 4 of 15

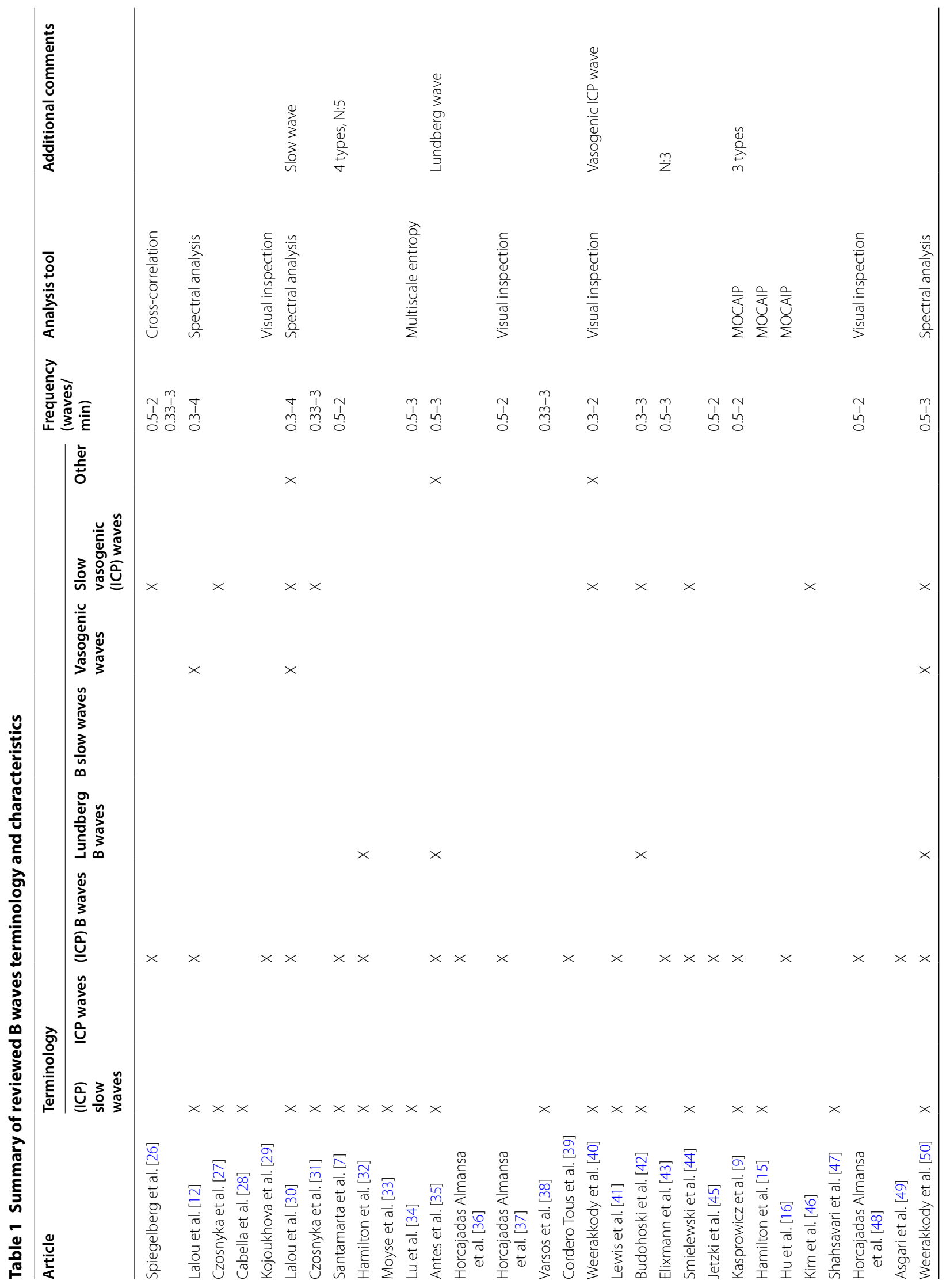




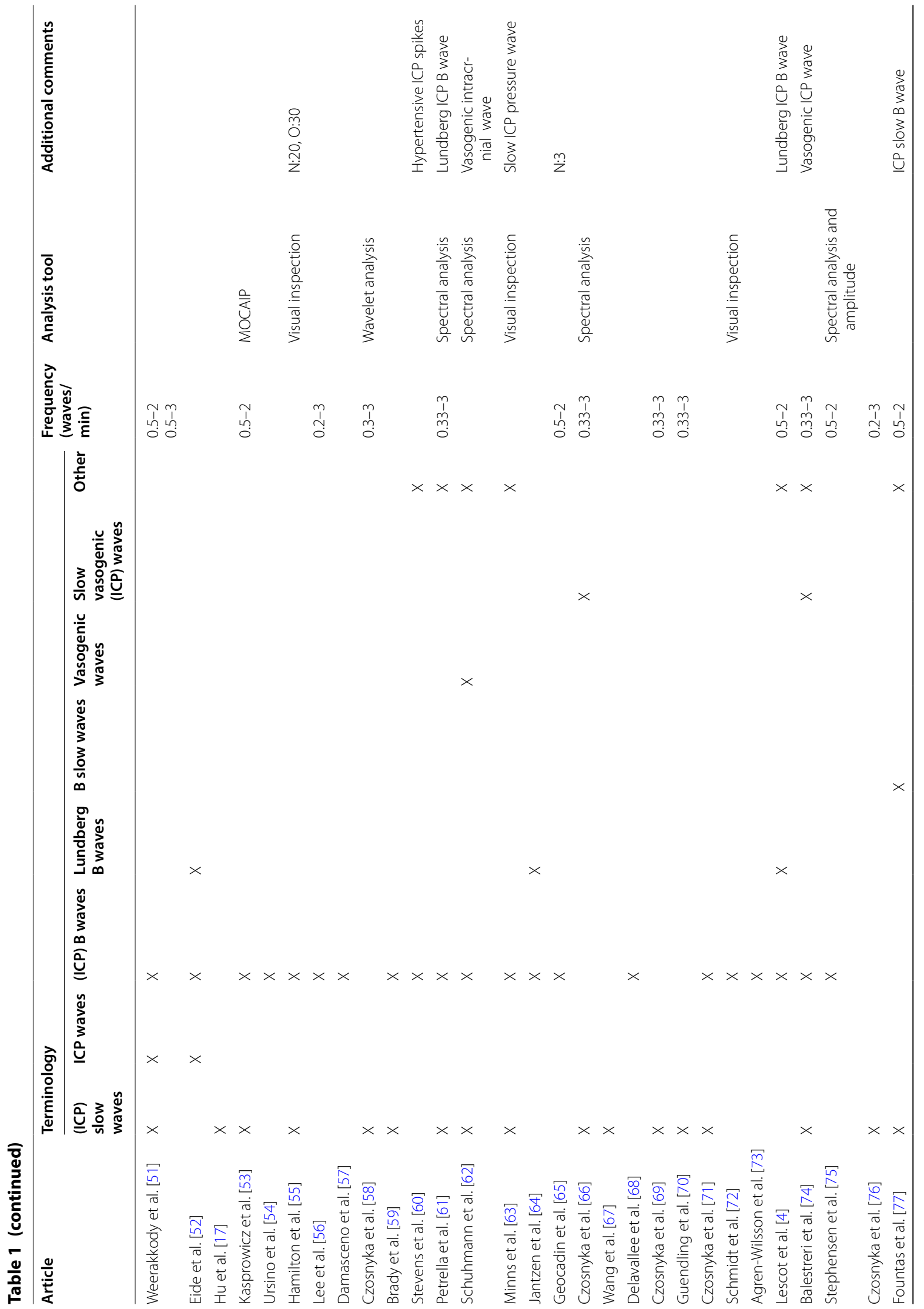




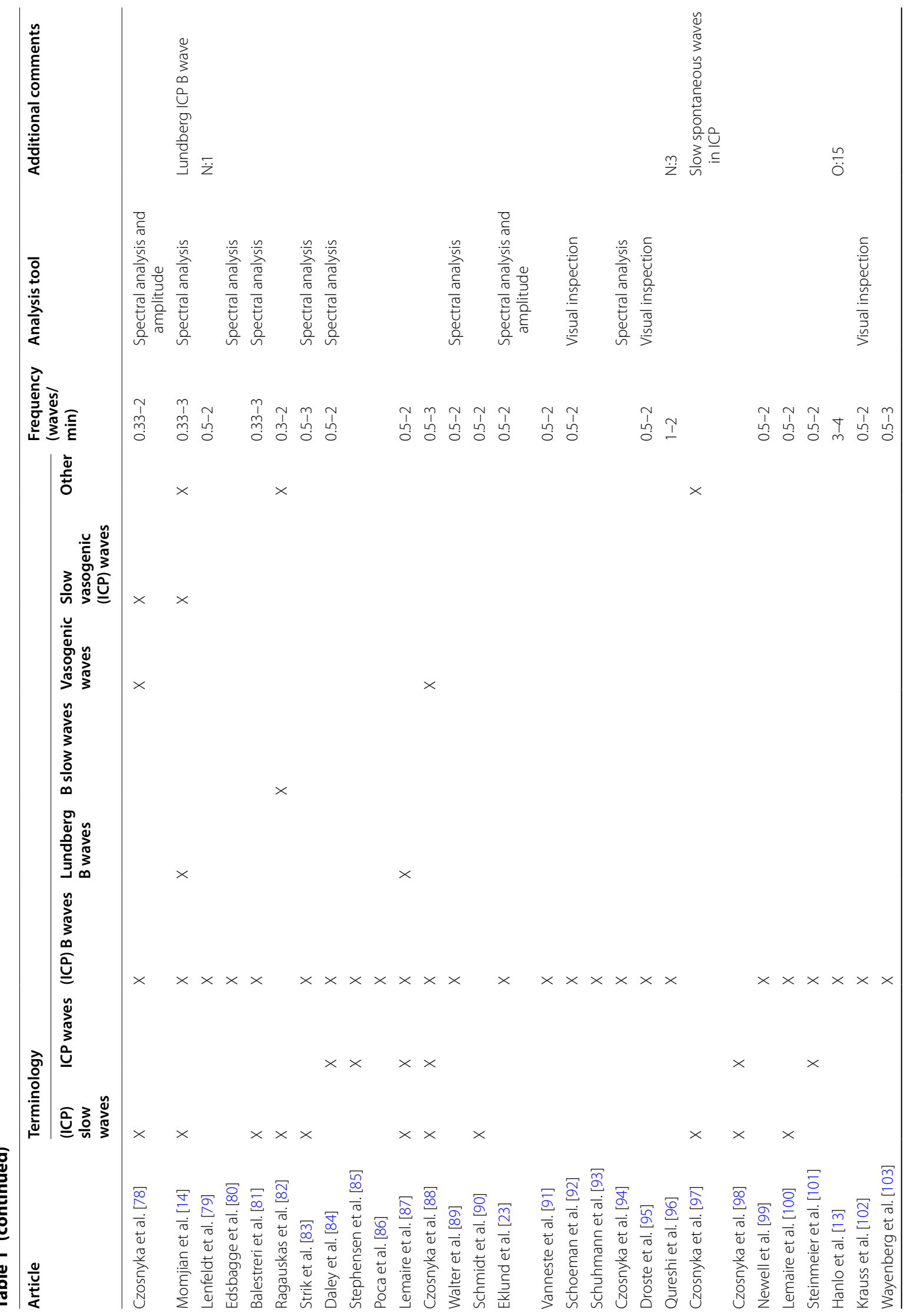


Martinez-Tejada et al. Fluids Barriers CNS

(2019) 16:33

Page 7 of 15

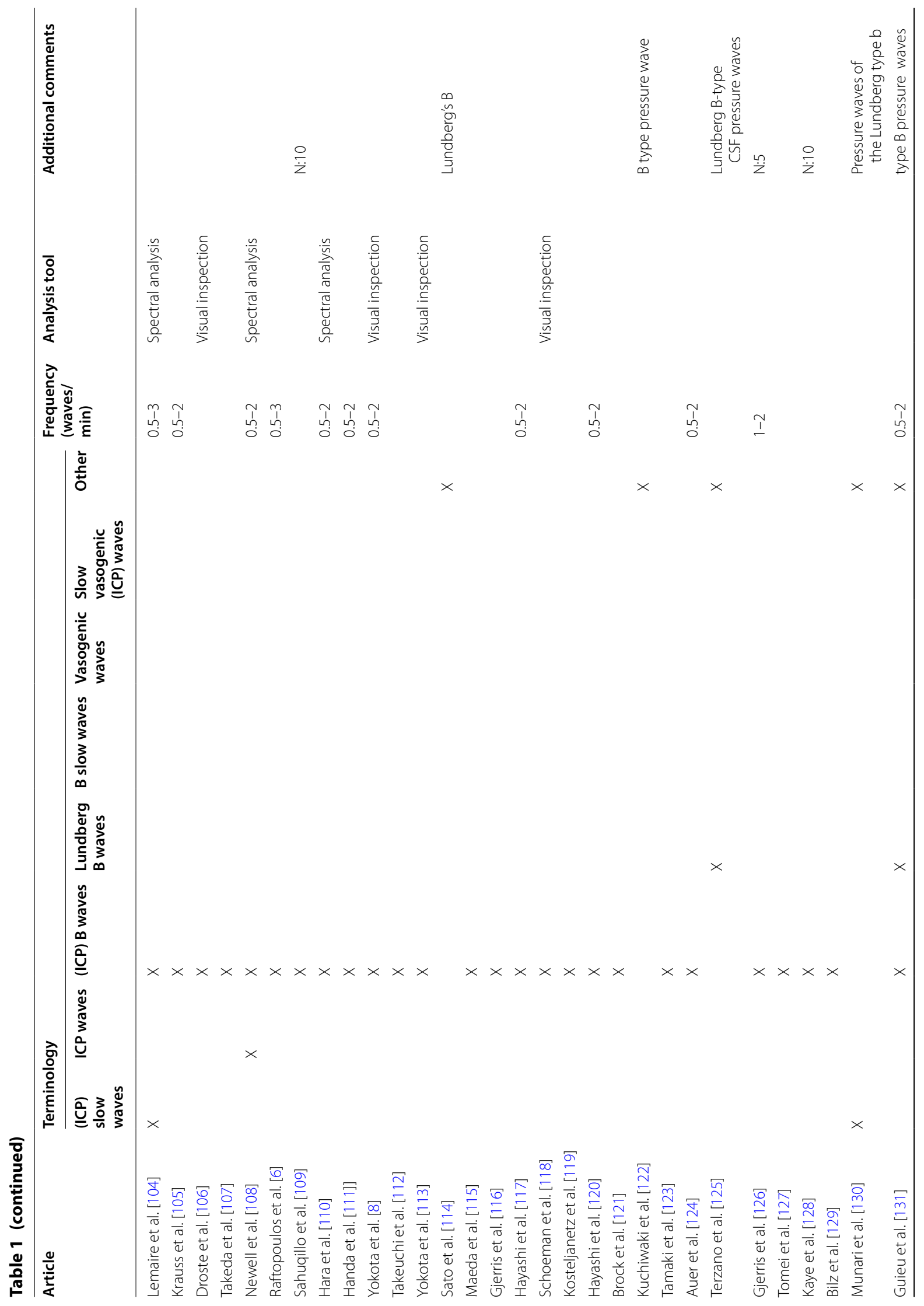




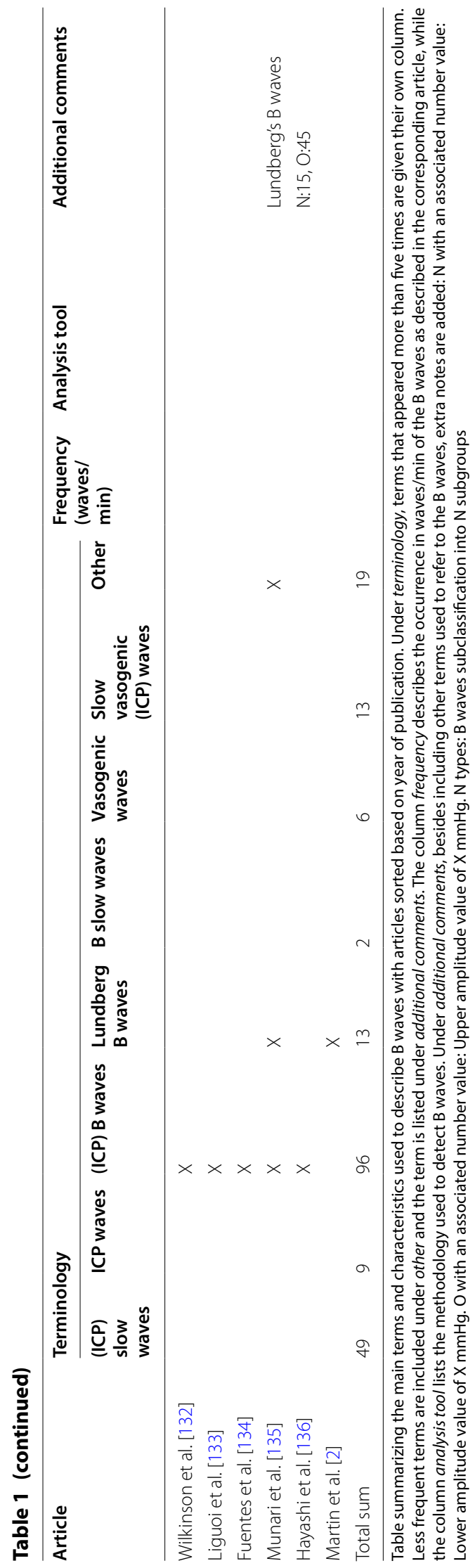




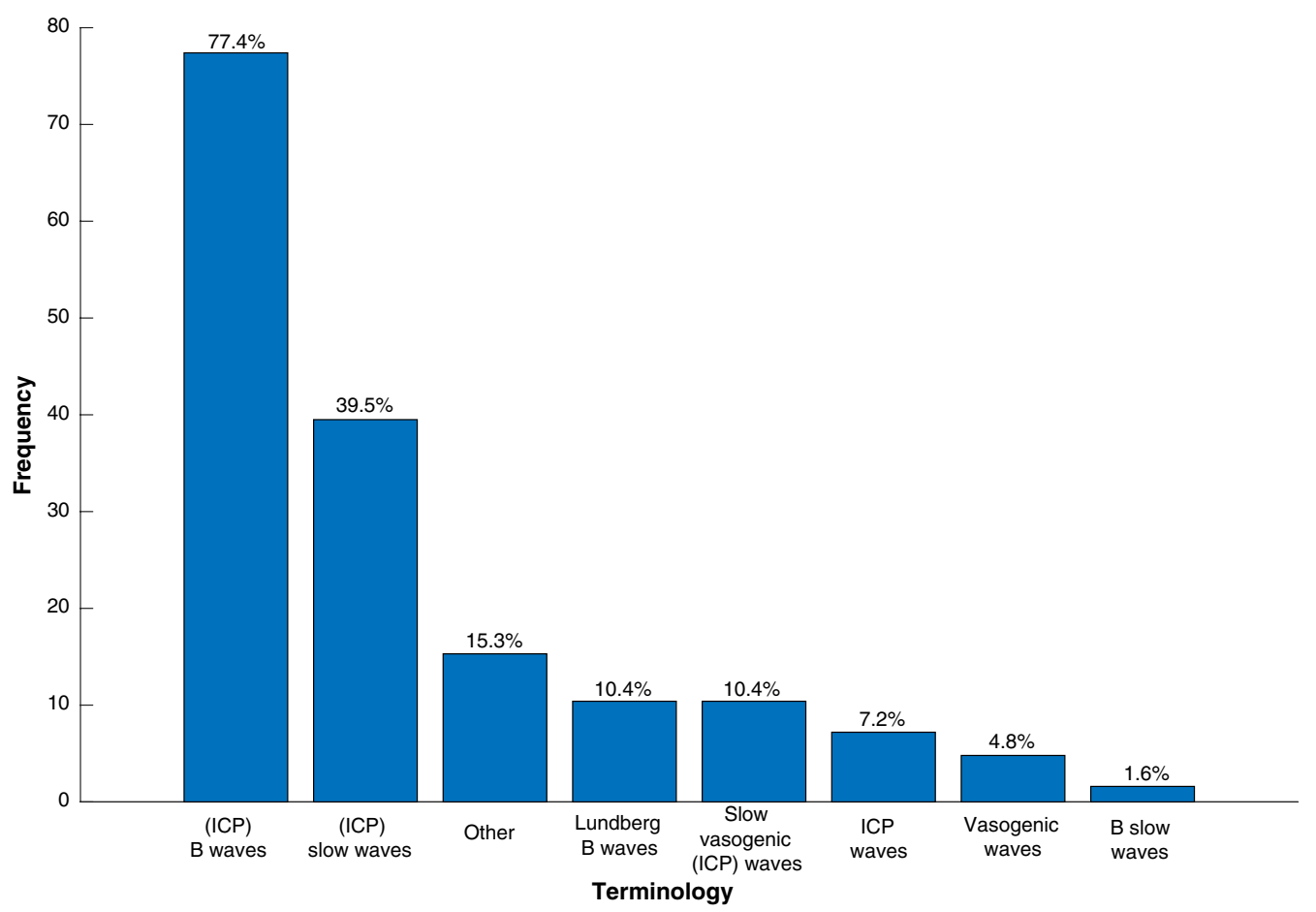

Fig. 2 Frequency of terminology usage in the reviewed papers. The term B waves was used in most articles, followed by slow waves and ICP slow waves

Table 2 Major morphological B wave subclasses

\begin{tabular}{|c|c|c|c|c|c|}
\hline & Term & Shape & Plateau & $\begin{array}{l}\text { Frequency } \\
\text { (waves/min) }\end{array}$ & $\begin{array}{l}\text { Amplitude } \\
(\mathrm{mmHg})\end{array}$ \\
\hline \multirow{3}{*}{$\begin{array}{l}\text { Raftopolous et al. [6] } \\
\text { Santamarta et al. [7] }\end{array}$} & Small symmetrical wave (SSW) & Symmetrical & No & $0.36-5$ & $<10$ \\
\hline & Great symmetrical wave (GSW) & Symmetrical & No & $0.36-5$ & $>10$ \\
\hline & Intermediate wave (IW) & Asymmetrical & No & $0.33-1.67$ & $6-34$ \\
\hline \multirow[t]{3}{*}{ Kasprowicz et al. [9] } & Slow symmetrical ICP wave & Symmetrical & No & - & - \\
\hline & Slow asymmetrical ICP wave & Asymmetrical & No & - & - \\
\hline & Slow ICP B with plateau phase & Symmetrical & Yes & - & - \\
\hline \multirow[t]{3}{*}{ Yokota et al. [8] } & Type II episodic B-wave & - & - & - & $25-75$ \\
\hline & Type III persistent, high pressure B-wave & - & - & $0.5-2$ & $40-100$ \\
\hline & Type IV continuous, regular B-wave & - & - & $0.5-2$ & $10-30$ \\
\hline
\end{tabular}

\section{Analysis tools}

$32 \%$ of the papers had an explicitly stated analysis method. While traditionally the most common analytical method used was either spectral analysis (40\%) or spectral analysis with an amplitude threshold (7\%), there is now an increasing tendency (10\%) to detect B waves using trained machine learning algorithms, as observed in more recently published papers $[9,15,16]$. These algorithms use as input morphological features extracted from the ICP pulse wave via the Morphological clustering and analysis of ICP pulse (MOCAIP) algorithm. Thus, instead of defining B waves in terms of amplitude and frequency, they define them according to different morphological parameters of the pulse wave. These parameters are based on the three subpeaks $\left(P_{1}, P_{2}\right.$, and $\left.P_{3}\right)$ of the pulse wave: systolic peak, tidal peak, and dicrotic peak, respectively [9]. Examples of these ICP pulse metrics include the amplitude of the subpeaks, the latency between subpeaks, and the start of the ICP pulse wave and the pulse wave period, among others [17]. 


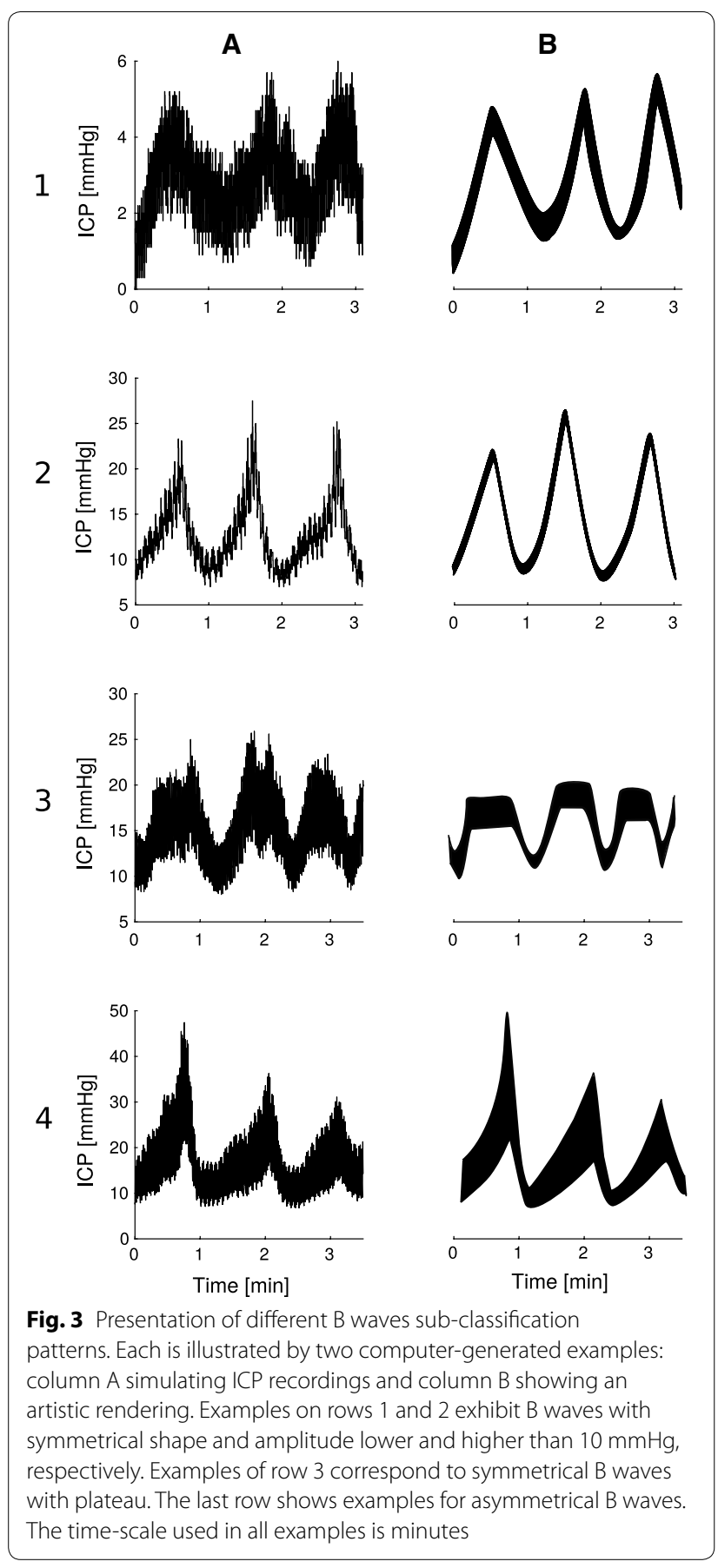

\section{Discussion}

ICP arises from pressure contributions from the brain, the heart, and the cerebrospinal fluid (CSF) inside the skull [18]. ICP is monitored invasively with a pressure transducer inserted either intracranially (subdural, epidural, intraparenchymal or intraventricular placements) or in the spinal compartment (lumbar puncture). As the brain is enclosed within the skull and its expandability is restricted, the ability to compensate for pressure-volume changes (auto-regulation) is also limited (i.e. compliance is low). Under normal conditions, auto-regulatory processes are responsible for keeping the intracranial volume constant. As brain compliance starts to decrease, the compensatory capacity is exhausted so that further volume changes are no longer accommodated; this causes ICP to increase. Space-occupying lesions are the main causes for the changes in intracranial volume. Hydrocephalus, intracranial haemorrhage, haematoma, and brain edema are examples of such lesions [19].

Under normal compensatory adaptations, the ICP stays within a narrow pressure range for each assumed body posture $[20,21]$. This is the simplest way of looking at ICP, as just a number that should remain within certain boundary values. Going beyond that, the ICP signal can be analyzed from a different perspective by studying the presence of macro-patterns. The diversity of $\mathrm{B}$ waves is the most commonly encountered macro-wave in clinical practice.

This study demonstrates the lack of agreement with regard to the terminology and characteristics used to define B waves. Different names are used to refer to the same phenomena, in order to either describe characteristics and morphological variations of the wave or the etiology behind their occurrence. This makes mathematical modeling of B waves more difficult, which consequently complicates the selection or development of an analysis tool that could be used to automatically interpret them. Automating B wave identification may be a way to detect and better understand ICP deviations from a normal physiological state at an earlier stage. But with the focus of current analysis tools on identifying previously defined B waves, they share a limitation of throwing away data related to other potentially relevant waveform deviations. Thus, underlying patterns of ICP that may contain important information on the interplay of physiological systems affecting the brain are potentially neglected. Opening up the analysis of ICP signals without being limited to previously defined patterns and conventions could enable fruitful new investigative and diagnostic techniques.

\section{Sub-classification}

$B$ waves were first defined from ICP monitoring sessions recorded in severely ill patients. Sub-classifications, which have mainly been qualitative, are the only attempts at modernizing the description of $\mathrm{B}$ waves to fit the clinical situations we see today [6-9].

The existence of multiple attempts at B wave subgrouping suggests that the overarching $B$ wave 
category is not satisfactory for classification purposes today. A future avenue of research may instead be to direct attention away from classical B wave detection and instead focus on the identification of new parameters to automate the analysis of repeatable patterns in the ICP signal. Pattern recognition algorithms will be the fundamental approach used for this purpose.

\section{Analysis tools}

ICP signals arise from the interaction of multiple physiological factors (e.g. heart pump, respiration, ...) that vary over time. Thus, it may be seen as a time series signal [22]. Traditionally, ICP signals have been inspected visually for B wave identification. In addition to being a time consuming technique, it is also subject to investigator bias due to interpretation subjectivity and dependence on clinical experience. Since the introduction of computerized algorithms, spectral analysis has led the way in $B$ wave detection. A general agreement on a certain frequency range that this wave occupies may explain why spectral analysis is the most reported methodology. However, there is low frequency activity within the B-wave range that is unrelated to vasomotor activity (i.e. respiratory changes associated with sleep), thereby introducing a severe limitation in the use of spectral analysis. We might get unwanted contributions from these signals in the B-wave frequency range when breaking down the signal into frequency components. Eklund et al. developed an algorithm that strives to overcome this problem by also taking into account the wave amplitude [23].

Defining B waves in terms of amplitude is, however, very ambiguous. In particular, the term amplitude can be approached as the trough to peak pressure difference in the signal. If the wave has a sinusoidal appearance there is no problem in the identification of both its maximum and minimum values, but their identification becomes a challenge when the waveform is irregular. At the same time, the term amplitude can also refer to the distance from the peak of the wave to the baseline.

MOCAIP extracts morphological parameters from the pulse wave that are then used to characterize $B$ waves instead of defining them based on their amplitude and frequency [24]. With the advantage of no longer depending on the classical B wave definition, this algorithm presents other drawbacks that prevents it from proper implementation in clinical practice. It rejects ICP pulses if a corresponding matching template is not included within the reference library proposed. This library is limited to intraparenchymal ICP signals from patients with hydrocephalus and does not comprise any ICP pulses from other pathologies, so that ICP pulses could be falsely rejected. Another limitation is the requirement of a simultaneous acquisition of ECG signal to help in the identification of the ICP pulse wave. Also, identifying $B$ waves using MOCAIP assumes that the pulse waves are affected during the B waves, which is not definitively settled. Another approach proposed by Elixmann also isolates the pulse waves and classifies them based on predefined templates [25].

\section{Conclusion}

To exploit the potential role of macro-patterns in ICP dynamics and to automate their identification for diagnostic or therapeutic purposes, two approaches for future work may be considered.

There could be efforts to arrive at strict agreement on morphological characteristics of classical macropatterns, which requires consensus-based definitions to enable the derivation of relevant metrics to characterize them.

Alternatively, a new approach could be attempted without relying on classical macro-patterns. Instead it could be based on recognition of new patterns that more adequately describe variations seen in daily clinical practice today. This de novo pattern recognition approach requires relating macro-patterns to clinical information to ensure that they are biologically relevant.

\section{Abbreviations \\ ICP: intracranial pressure; CSF: cerebrospinal fluid; ICU: intensive care unit; MOCAIP: morphological clustering and analysis of ICP pulse.}

\section{Acknowledgements}

The authors are thankful for contributions from the Novo Nordisk Fonden Tandem Programme.

\section{Authors' contributions}

Study conception and design: AA, MA, MJ. Analysis and interpretation of the papers for the work: AA and IMT. Drafting of manuscript: IMT with input from the other authors. Critical revision: MJ, MA, JW. All authors read and approved the final manuscript.

\section{Funding}

Funding was provided by Novo Nordisk Fonden (Grant Number: NNF17OC0024718)

Availability of data and materials

Data sharing is not applicable to this article as no datasets were generated or analysed during the current study.

Ethics approval and consent to participate

Not applicable.

Consent for publication

Not applicable.

Competing interests

The authors declare that they have no competing interests. 


\section{Author details}

${ }^{1}$ Clinic of Neurosurgery, Copenhagen University Hospital, Rigshospitalet, Copenhagen, Denmark. ${ }^{2}$ Department of Health Technology, Technical University of Denmark, Kongens Lyngby, Denmark.

Received: 12 June 2019 Accepted: 15 September 2019 Published online: 15 October 2019

\section{References}

1. Lundberg N. Continuous recording and control of ventricular fluid pressure in neurosurgical practice. Acta Psychiatrica Scandinavica Supplementum. 1960;36:1-193.

2. Martin G. Lundberg's B waves as a feature of normal intracranial pressure. Surg Neurol. 1978;9(6):347-8.

3. Droste DW, Krauss JK, Berger W, Schuler E, Brown MM. Rhythmic oscillations with a wavelength of 0.5-2 min in transcranial Doppler recordings. Acta Neurologica Scandinavica. 2009;90(2):99-104.

4. Lescot T, Naccache L, Bonnet MP, Abdennour L, Coriat P, Puybasset L. The relationship of intracranial pressure Lundberg waves to electroencephalograph fluctuations in patients with severe head trauma. Acta Neurochir. 2005;147(2):125-9 (discussion 129)

5. Liberati A, Altman DG, Tetzlaff J, Mulrow C, Gotzsche PC, loannidis JPA, et al. The PRISMA statement for reporting systematic reviews and metaanalyses of studies that evaluate healthcare interventions: explanation and elaboration. Br Med J. 2009;339(b2700):b2700.

6. Raftopoulos C, Chaskis C, Delecluse F, Cantrainet F, Bidauti L, Brotchi J. Morphological quantitative analysis of intracranial pressure waves in normal pressure hydrocephalus. Neurol Res. 1992;14:389-96.

7. Santamarta D, González-Martínez E, Fernández J, Mostaza A. The prediction of shunt response in idiopathic normal-pressure hydrocephalus based on intracranial pressure monitoring and lumbar infusion. Acta Neurochir Suppl. 2016;122:267-74.

8. Yokota A, Matsuoka S, Ishikawa T, Kohshi K, Kajiwara H. Overnight recordings of intracranial pressure and electroencephalography in neurosurgical patients. Part I: intracranial pressure waves and their clinical correlations. J UOEH. 1989;11(4):371-81.

9. Kasprowicz M, Bergsneider M, Czosnyka M, Hu X. Association between ICP pulse waveform morphology and ICP B waves. Acta Neurochir Suppl. 2012;114:29-34.

10. Spiegelberg A, Preuß M, Kurtcuoglu V. B-waves revisited. Interdisciplin Neurosurgery: Adv Tech Case Manage. 2016;6:13-7.

11. Czosnyka M, Pickard JD. Monitoring and interpretation of intracranial pressure. J Neurol Neurosurg Psychiatry. 2004;75(6):813-21.

12. Lalou DA, Czosnyka M, Donnelly J, Lavinio A, Pickard JD, Garnett M, et al. Are slow waves of intracranial pressure suppressed by general anaesthesia? Acta Neurochir Suppl. 2018;126:129-32.

13. Hanlo PW, Gooskens RH, Faber JA, Peters RJ, Hermsen AA, Nijhuis IJ, et al. Relationship between anterior fontanelle pressure measurements and clinical signs in infantile hydrocephalus. Childs Nerv Syst. 1996;12(4):200-9.

14. Momjian S, Czosnyka Z, Czosnyka M, Pickard JD. Link between vasogenic waves of intracranial pressure and cerebrospinal fluid outflow resistance in normal pressure hydrocephalus. Br J Neurosurg. 2004;18(1):56-61.

15. Hamilton R, Baldwin K, Fuller J, Vespa P, Hu X, Bergsneider M. Intracranial pressure pulse waveform correlates with aqueductal cerebrospinal fluid stroke volume. J Appl Physiol. 2012;113(10):1560-6.

16. Hu X, Hamilton R, Baldwin K, Vespa PM, Bergsneider M. Automated extraction of decision rules for predicting lumbar drain outcome by analyzing overnight intracranial pressure. Acta Neurochir Suppl. 2012;114:207-12.

17. Hu X, Xu P, Asgari S, Vespa P, Bergsneider M. Forecasting ICP elevation based on prescient changes of intracranial pressure waveform morphology. IEEE Trans Biomed Eng. 2010;57(5):1070-8.

18. Raboel PH, Bartek J, Andresen M, Bellander BM, Romner B. Intracranial pressure monitoring: Invasive versus non-invasive methods - a review. Crit Care Res Pract. 2012;2012:950393.
19. Lavinio A, Menon DK. Intracranial pressure: why we monitor it, how to monitor it, what to do with the number and what's the future? Curr Opin Anesthesiol. 2011:24:117-23.

20. Andresen $M$, Hadi $A$, Petersen $L G$, Juhler $M$. Effect of postural changes on ICP in healthy and ill subjects. Acta Neurochir. 2014;157(1):109-13.

21. Petersen LG, Lawley JS, Lilja-Cyron A, Petersen JCG, Howden EJ, Sarma S, et al. Lower body negative pressure to safely reduce intracranial pressure. J Physiol. 2019;597(1):237-48.

22. Pham H. Springer handbook of engineering statistics. London: Springer; 2006.

23. Eklund A, Agren-Wilsson A, Andersson N, Bergenheim AT, Koskinen LO, Malm J. Two computerized methods used to analyze intracranial pressure B waves: comparison with traditional visual interpretation. J Neurosurg. 2001;94(3):392-6.

24. Hu X, Xu P, Scalzo F, Vespa P, Bergsneider M. Morphological clustering and analysis of continuous intracranial pressure. IEEE Trans Biomed Eng. 2009;56(4667641):696-705.

25. Elixmann IM, Hansinger J, Goffin C, Antes S, Radermacher K, Leonhardt S. Single pulse analysis of intracranial pressure for a hydrocephalus implant. In: Proceedings of the annual international conference of the IEEE engineering in medicine and biology Society, Embs, vol. 2012, no. 6346828. 2012. p. 3939-42.

26. Spiegelberg A, Krause M, Meixensberger J, Seifert B, Kurtcuoglu V. Significant association of slow vasogenic ICP waves with normal pressure hydrocephalus diagnosis. Acta Neurochir Suppl. 2018;126:243-6.

27. Czosnyka M, Donnelly J, Calviello L, Smielewski P, Menon DK, Pickard JD. Do ICP-derived parameters differ in vegetative state from other outcome groups after traumatic brain injury? Acta Neurochir Suppl. 2018;126:17-20.

28. Cabella B, Donnelly J, Cardim D, Liu X, Cabeleira M, Smielewski P, et al. An association between ICP-derived data and outcome in TBI patients: the role of sample size. Neurocrit Care. 2017;27(1):103-7.

29. Kojoukhova M, Vanha Kl, Timonen M, Koivisto AM, Nerg O, Rummukainen J, et al. Associations of intracranial pressure with brain biopsy, radiological findings, and shunt surgery outcome in patients with suspected idiopathic normal pressure hydrocephalus. Acta Neurochir. 2017;159(1):51-61.

30. Lalou DA, Czosnyka M, Donnelly J, Lavinio A, Pickard JD, Garnett M, et al. Influence of general anaesthesia on slow waves of intracranial pressure. Neurol Res. 2016;38(7):587-92.

31. Czosnyka M, Varsos GV, Czosnyka ZH, Smielewski P, Saadoun S, Jamous A, et al. Waveform analysis of intraspinal pressure after traumatic spinal cord injury: an observational study (O-64). Acta Neurochir Suppl. 2016;122:335-8.

32. Hamilton R, Fuller J, Baldwin K, Vespa P, Hu X, Bergsneider M. Relative position of the third characteristic peak of the intracranial pressure pulse waveform morphology differentiates normal-pressure hydrocephalus shunt responders and nonresponders. Acta Neurochir Suppl. 2016;122:339-45.

33. Moyse E, Ros M, Marhar F, Swider P, Schmidt EA. Characterisation of supra- and infratentorial ICP profiles. Acta Neurochir Suppl. 2016;122:37-40.

34. Lu CW, Czosnyka M, Shieh JS, Pickard JD, Smielewski P. Continuous monitoring of the complexity of intracranial pressure after head injury. Acta Neurochir Suppl. 2016;122:33-5.

35. Antes S, Tschan CA, Heckelmann M, Breuskin D, Oertel J. Telemetric intracranial pressure monitoring with the Raumedic Neurovent P-tel. World Neurosurg. 2016;91:133-48.

36. Horcajadas Almansa A, Cordero Tous N, Roman Cutillas A, Jorques Infante A, Saura Rojas E, Ibanez Velasco B, et al. Usefulness of continuous intracranial pressure monitoring in long-standing overt ventriculomegaly in adults. Neurocirugia. 2015;26(2):64-72.

37. Horcajadas Almansa A, Cordero Tous N, Roman Cutillas A, Jorques Infante A, Saura Rojas E, Ibanez Velasco B, et al. Usefulness of intracranial pressure continuous monitoring in pseudotumor cerebri. Neurocirugia. 2015;26(4):157-66.

38. Varsos GV, Werndle MC, Czosnyka ZH, Smielewski P, Kolias AG, Phang I, et al. Intraspinal pressure and spinal cord perfusion pressure after spinal cord injury: an observational study. J Neurosurg Spine. 2015:23(6):763-71. 
39. Cordero Tous N, Roman Cutillas AM, Jorques Infante AM, Olivares Granados G, Saura Rojas JE, Ibanez Velasco B, et al. Adult chronic idiopathic hydrocephalus-diagnosis, treatment and evolution. Prospective study. Neurocirugia. 2013;24(3):93-101.

40. Weerakkody RA, Czosnyka M, Zweifel C, Castellani G, Smielewski P, Brady K, et al. Near infrared spectroscopy as possible non-invasive monitor of slow vasogenic ICP waves. Acta Neurochir Suppl. 2012;114:181-5.

41. Lewis PM, Smielewski P, Rosenfeld JV, Pickard JD, Czosnyka M. Monitoring of the association between cerebral blood flow velocity and intracranial pressure. Acta Neurochir Suppl. 2012;114:147-51.

42. Budohoski KP, Schmidt B, Smielewski P, Kasprowicz M, Plontke R, Pickard JD, et al. Non-invasively estimated ICP pulse amplitude strongly correlates with outcome after TBI. Acta Neurochir Suppl. 2012;114:121-5.

43. Elixmann IM, Walter $M$, Kiefer $M$, Leonhardt S. Simulation of existing and future electromechanical shunt valves in combination with a model for brain fluid dynamics. Acta Neurochir Suppl. 2012;113:77-81.

44. Smielewski P, Czosnyka Z, Kasprowicz M, Pickard JD, Czosnyka M. ICM+: a versatile software for assessment of CSF dynamics. Acta Neurochir Suppl. 2012;114:75-9.

45. Jetzki S, Weinzierl M, Krause I, Hahne S, Rehbaum H, Kiausch M, et al. A multisensor implant for continuous monitoring of intracranial pressure dynamics. IEEE Trans Biomed Circuits Syst. 2012;6(4):356-65.

46. Kim DJ, Czosnyka Z, Kasprowicz M, Smieleweski P, Baledent O, Guerguerian AM, et al. Continuous monitoring of the Monro-Kellie doctrine: is it possible? J Neurotrauma. 2012;29(7):1354-63.

47. Shahsavari S, McKelvey T, Ritzén CE, Rydenhag B. Cerebrovascular mechanical properties and slow waves of intracranial pressure in TB patients. IEEE Trans Biomed Eng. 2011;58(7):2072-82.

48. Horcajadas Almansa A, Roman A, Olivares G, Saura E, Jorques A, Cordero $\mathrm{N}$, et al. Usefulness of IPC continuous monitoring in shunt dysfunction. Neurocirugia. 2011;22(4):310-23.

49. Asgari S, Vespa P, Bergsneider M, Hu X. Lack of consistent intracrania pressure pulse morphological changes during episodes of microdialysis lactate/pyruvate ratio increase. Physiol Meas. 2011;32(10):1639-51.

50. Weerakkody RA, Czosnyka M, Schuhmann MU, Schmidt E, Keong N, Santarius T, et al. Clinical assessment of cerebrospinal fluid dynamics in hydrocephalus. Guide to interpretation based on observational study. Acta Neurol Scand. 2011;124(2):85-98.

51. Weerakkody RA, Czosnyka M, Zweifel C, Castellani G, Smielewski P, Keong $\mathrm{N}$, et al. Slow vasogenic fluctuations of intracranial pressure and cerebral near infrared spectroscopy — an observational study. Acta Neurochir. 2010;152(10):1763-9.

52. Eide PK, Sorteberg W. Simultaneous measurements of intracranial pressure parameters in the epidural space and in brain parenchyma in patients with hydrocephalus. J Neurosurg. 2010;113(6):1317-25.

53. Kasprowicz M, Asgari S, Bergsneider M, Czosnyka M, Hamilton R, Hu $X$. Pattern recognition of overnight intracranial pressure slow waves using morphological features of intracranial pressure pulse. J Neurosci Methods. 2010;190(2):310-8

54. Ursino M, Giannessi M, Frapparelli M, Magosso E. Effect of cushing response on systemic arterial pressure. IEEE Eng Med Biol Mag. 2009;28(6):63-71.

55. Hamilton R, Xu P, Asgari S, Kasprowicz M, Vespa P, Bergsneider M, et al. Forecasting intracranial pressure elevation using pulse waveform morphology. Conf Proc IEEE Eng Med Biol Soc. 2009;2009:4331-4.

56. Lee JK, Kibler KK, Benni PB, Easley RB, Czosnyka M, Smielewski P, et al. Cerebrovascular reactivity measured by near-infrared spectroscopy. Stroke. 2009;40(5):1820-6.

57. Damasceno BP. Normal pressure hydrocephalus: diagnostic and predictive evaluation. Dement Neuropsychol. 2009;3(1):8-15.

58. Czosnyka M, Brady K, Reinhard M, Smielewski P, Steiner LA. Monitoring of cerebrovascular autoregulation: facts, myths, and missing links. Neurocrit Care. 2009;10(3):373-86.

59. Brady KM, Lee JK, Kibler KK, Easley RB, Koehler RC, Shaffner DH. Continuous measurement of autoregulation by spontaneous fluctuations in cerebral perfusion pressure: comparison of 3 methods. Stroke. 2008:39(9):2531-7.
60. Stevens SA, Stimpson J, Lakin WD, Thakore NJ, Penar PL. A model for idiopathic intracranial hypertension and associated pathological ICP wave-forms. IEEE Trans Biomed Eng. 2008;55(2 Pt 1):388-98.

61. Petrella G, Czosnyka M, Keong N, Pickard JD, Czosnyka Z. How does CSF dynamics change after shunting? Acta Neurol Scand. 2008;118(3):182-8.

62. Schuhmann MU, Sood S, McAllister JP, Jaeger M, Ham SD, Czosnyka Z et al. Value of overnight monitoring of intracranial pressure in hydrocephalic children. Pediatr Neurosurg. 2008;44(4):269-79.

63. Minns RA, Jones PA, Chambers IR. Low frequency pressure waves of possible autonomic origin in severely head-injured children. Acta Neurochir Suppl. 2008;102:85-8.

64. Jantzen JPAH. Prevention and treatment of intracranial hypertension. Best Pract Res Clin Anaesthesiol. 2007:21(4):517-38.

65. Geocadin RG, Varelas PN, Rigamonti D, Williams MA. Continuous intracranial pressure monitoring via the shunt reservoir to assess suspected shunt malfunction in adults with hydrocephalus. Neurosurg Focus. 2007;22(4):E10.

66. Czosnyka M, Smielewski P, Timofeev I, Lavinio A, Guazzo E, Hutchinson $P$, et al. Intracranial pressure: more than a number. Neurosurg Focus. 2007;22(5):E10.

67. Wang EC, Ang BT, Wong J, Lim J, Ng I. Characterization of cerebrovascular reactivity after craniectomy for acute brain injury. Br J Neurosurg 2006;20(1):24-30

68. Delavallée M, Raftopoulos C. Normal pressure hydrocephalus in a patient with myotonic dystrophy: case report with a 10-year follow-up. Neurosurgery. 2006;58(4):E796 (discussion E796)

69. Czosnyka M, Hutchinson PJ, Balestreri M, Hiler M, Smielewski P, Pickard JD. Monitoring and interpretation of intracranial pressure after head injury. Acta Neurochir Suppl. 2006;96:114-8.

70. Guendling K, Smielewski P, Czosnyka M, Lewis P, Nortje J, Timofeev I, et al. Use of ICM+ software for on-line analysis of intracranial and arterial pressures in head-injured patients. Acta Neurochir Suppl. 2006;96:108-13.

71. Czosnyka Z, van den Boogaard F, Czosnyka M, Momjian S, Gelling L, Pickard JD. The relationship between CSF circulation and cerebrovascular pressure-reactivity in normal pressure hydrocephalus. Acta Neurochir Suppl. 2005:95:207-11.

72. Schmidt B, Bocklisch SF, Pässler M, Czosnyka M, Schwarze JJ, Klingelhöfer J. Fuzzy pattern classification of hemodynamic data can be used to determine noninvasive intracranial pressure. Acta Neurochir Suppl. 2005:95:345-9.

73. Agren-Wilsson A, Eklund A, Koskinen LOD, Bergenheim AT, Malm J. Brain energy metabolism and intracranial pressure in idiopathic adult hydrocephalus syndrome. J Neurol Neurosurg Psychiatry. 2005;76(8):1088-93.

74. Balestreri M, Czosnyka M, Steiner LA, Hiler M, Schmidt EA, Matta B, et al. Association between outcome, cerebral pressure reactivity and slow ICP waves following head injury. Acta Neurochir Suppl. 2005;95:25-8.

75. Stephensen $H$, Andersson N, Eklund A, Malm J, Tisell M, Wikkelsö C. Objective B wave analysis in 55 patients with non-communicating and communicating hydrocephalus. J Neurol Neurosurg Psychiatry. 2005;76(7):965-70.

76. Czosnyka M, Balestreri M, Steiner L, Smielewski P, Hutchinson PJ, Matta $B$, et al. Age, intracranial pressure, autoregulation, and outcome after brain trauma. J Neurosurg. 2005;102(3):450-4.

77. Fountas KN, Sitkauskas A, Feltes CH, Kapsalaki EZ, Dimopoulos VG, Kassam $M$, et al. Is non-invasive monitoring of intracranial pressure waveform analysis possible? Preliminary results of a comparative study of non-invasive vs. invasive intracranial slow-wave waveform analysis monitoring in patients with traumatic brain injury. Med Sci Monit. 2005;11(2):CR58-63.

78. Czosnyka M, Czosnyka Z, Momjian S, Pickard JD. Cerebrospinal fluid dynamics. Physiol Meas. 2004;25(5):R51-76.

79. Lenfeldt N, Andersson N, Agren-Wilsson A, Bergenheim AT, Koskinen LOD, Eklund A, et al. Cerebrospinal fluid pulse pressure method: a possible substitute for the examination of B waves. J Neurosurg. 2004;101(6):944-50

80. Edsbagge $M$, Tisell M, Jacobsson L, Wikkelso C. Spinal CSF absorption in healthy individuals. Am J Physiol Regul Integr Comp Physiol. 2004;287(6):R1450-5. 
81. Balestreri M, Czosnyka M, Steiner LA, Schmidt E, Smielewski P, Matta $B$, et al. Intracranial hypertension: what additional information can be derived from ICP waveform after head injury? Acta Neurochir. 2004;146(2):131-41

82. Ragauskas A, Daubaris G, Ragaisis V, Petkus V. Implementation of non-invasive brain physiological monitoring concepts. Med Eng Phys. 2003;25(8):667-78.

83. Strik C, Klose U, Kiefer C, Grodd W. Slow rhythmic oscillations in intracranial CSF and blood flow: registered by MRI. Acta Neurochir Suppl. 2002;81:139-42.

84. Daley ML, Pasley RL, Connolly M, Timmons SD, Angel J, Stidham G, et al. Spectral characteristics of B-waves and other low-frequency activity. Acta Neurochir Suppl. 2002;81:147-50.

85. Stephensen $\mathrm{H}$, Tisell $\mathrm{M}$, Wikkelsö $\mathrm{C}$. There is no transmantle pressure gradient in communicating or noncommunicating hydrocephalus. Neurosurgery. 2002;50(4):763-71 (discussion 771-773).

86. Poca MA, Sahuquillo J, Ibañez J, Amorós S, Arikan F, Rubio E. Intracranial hypertension after surgery in patients with Chiari I malformation and normal or moderate increase in ventricular size. Acta Neurochir Suppl. 2002;81:35-8.

87. Lemaire JJ, Khalil T, Cervenansky F, Gindre G, Boire JY, Bazin JE, et al. Slow pressure waves in the cranial enclosure. Acta Neurochir. 2002;144(3):243-54

88. Czosnyka ZH, Czosnyka M, Whitfield PC, Donovan T, Pickard JD. Cerebral autoregulation among patients with symptoms of hydrocephalus. Neurosurgery. 2002;50(3):526-32 (discussion 532-533).

89. Walter M, Kiefer M, Leonhardt S, Steudel WI, Isermann R. Online analysis of intracranial pressure waves. Acta Neurochir Suppl. 2002;81:161-2.

90. Schmidt EA, Czosnyka M, Smielewski P, Piechnik SK, Pickard JD. Asymmetry of cerebral autoregulation following head injury. Acta Neurochir Suppl. 2002;81:133-4.

91. Vanneste JA. Diagnosis and management of normal-pressure hydrocephalus. J Neurol. 2000;247(1):5-14

92. Schoeman JF, Laubscher JA, Donald PR. Serial lumbar CSF pressure measurements and cranial computed tomographic findings in childhood tuberculous meningitits. Childs Nerv Syst. 2000;16(4):203-8 (discussion 209)

93. Schuhmann MU, Engel M, Runge L, Samii M, Brinker T. Application of clinically recorded ICP patterns-an extension of conventional shunt testing. Childs Nerv Syst. 2000;16(12):856-61.

94. Czosnyka M, Smielewski P, Piechnik S, Al-Rawi PG, Kirkpatrick PJ, Matta BF, et al. Critical closing pressure in cerebrovascular circulation. J Neurol Neurosurg Psychiatry. 1999;66(5):606-11.

95. Droste DW, Krauss JK. Intracranial pressure B-waves precede corresponding arterial blood pressure oscillations in patients with suspected normal pressure hydrocephalus. Neurol Res. 1999;21(7):627-30.

96. Qureshi Al, Williams MA, Razumovsky AY, Hanley DF. Magnetic resonance imaging, unstable intracranial pressure and clinical outcome in patients with normal pressure hydrocephalus. Acta Neurochir Suppl. 1998;71:354-6.

97. Czosnyka M, Smielewski P, Kirkpatrick P, Piechnik S, Laing R, Pickard JD. Continuous monitoring of cerebrovascular pressure-reactivity in head injury. Acta Neurochir Suppl. 1998;71:74-7.

98. Czosnyka M, Smielewski P, Kirkpatrick P, Laing RJ, Menon D, Pickard JD. Continuous assessment of the cerebral vasomotor reactivity in head injury. Neurosurgery. 1997;41(1):11-7 (discussion 17-19).

99. Newell DW, Aaslid R, Stooss R, Seiler RW, Reulen HJ. Evaluation of hemodynamic responses in head injury patients with transcranial Doppler monitoring. Acta Neurochir. 1997;139(9):804-17.

100. Lemaire JJ. Slow pressure waves during intracranial hypertension. Ann Fr Anesth Reanim. 1997;16(4):394-8.

101. Steinmeier R, Bauhuf C, Hübner U, Bauer RD, Fahlbusch R, Laumer R, et al. Slow rhythmic oscillations of blood pressure, intracranial pressure, microcirculation, and cerebral oxygenation. Dynamic interrelation and time course in humans. Stroke. 1996;27(12):2236-43.

102. Krauss JK, Droste DW, Bohus M, Regel JP, Scheremet R, Riemann D, et al. The relation of intracranial pressure B-waves to different sleep stages in patients with suspected normal pressure hydrocephalus. Acta Neurochir. 1995;136(3-4):195-203.
103. Wayenberg $\mathrm{J}$, Hasaerts D, Franco P, Valente F, Massager N. Anterior fontanelle pressure variations during sleep in healthy infants. Sleep. 1995:18(4):223-8.

104. Lemaire JJ, Boire JY, Chazal J, Irthum B. A computer software for frequential analysis of slow intracranial pressure waves. Comput Methods Programs Biomed. 1994;42(1):1-14.

105. Krauss JK, Droste DW. Predictability of intracranial pressure oscillations in patients with suspected normal pressure hydrocephalus by transcranial Doppler ultrasound. Neurol Res. 1994;16(5):398-402.

106. Droste DW, Krauss JK, Berger W, Schuler E, Brown MM. Rhythmic oscillations with a wavelength of $0.5-2$ min in transcranial Doppler recordings. Acta Neurol Scand. 1994;90(2):99-104.

107. Takeda Y, Onitsuka T, Haraoka J, Koba T, Ito H, Miwa T. Clinical assessment of meningeal carcinomatosis; from the viewpoint of the analysis of intracranial pressure. No Shinkei Geka. 1993;21(3):213-20.

108. Newell DW, Aaslid R, Stooss R, Reulen HJ. The relationship of blood flow velocity fluctuations to intracranial pressure B waves. J Neurosurg. 1992;76(3):415-21.

109. Sahuquillo J, Rubio E, Codina A, Molins A, Guitart JM, Poca MA, et al. Reappraisal of the intracranial pressure and cerebrospinal fluid dynamics in patients with the so-called "normal pressure hydrocephalus" syndrome. Acta Neurochir. 1991;112(1-2):50-61.

110. Hara K, Nakatani S, Ozaki K, Ikeda T, Mogami H. Detection of the B waves in the oscillation of intracranial pressure by fast Fourier transform. Med Inform. 1990;15(2):125-31.

111. Handa Y, Hayashi M, Hirose S, Noguchi Y, Kobayashi H. The effect of increased intracranial pressure during the appearance of pressure waves on the brainstem. Neurol Med Chir. 1990;30(5):301-8.

112. Takeuchi S, Koike T, Sasaki O, Kamada K, Tanaka R, Arai H. Intracranial extradural pressure monitoring after direct operation on ruptured cerebral aneurysms. Neurosurgery. 1989;24(6):878-83.

113. Yokota A, Matsuoka S, Ishikawa T, Kohshi K, Kajiwara H. Overnight recordings of intracranial pressure and electroencephalography in neurosurgical patients. Part II: changes in intracranial pressure during sleep. J UOEH. 1989;11(4):383-91.

114. Sato H, Sato N, Tamaki N, Matsumoto S. The critical threshold of cerebral perfusion pressure in intracranial pressure circumstance of hydrocephalus during infancy. No Shinkei Geka. 1988;16(4):385-92.

115. Maeda M, Matsuura S, Tanaka K, Katsuyama J, Nakamura T, Sakamoto $\mathrm{H}$, et al. Effects of electrical stimulation on intracranial pressure and systemic arterial blood pressure in cats. Part I: stimulation of brain stem. Neurol Res. 1988;10(2):87-92.

116. Gjerris F, Børgesen SE, Sørensen PS, Boesen F, Schmidt K, Harmsen A, et al. Resistance to cerebrospinal fluid outflow and intracranial pressure in patients with hydrocephalus after subarachnoid haemorrhage. Acta Neurochir. 1987;88(3-4):79-86.

117. Hayashi M, Kobayashi H, Kawano H, Handa Y, Yamamoto S, Kitano T. ICP patterns and isotope cisternography in patients with communicating hydrocephalus following rupture of intracranial aneurysm. J Neurosurg. 1985;62(2):220-6

118. Schoeman JF, le Roux D, Bezuidenhout PB, Donald PR. Intracranial pressure monitoring in tuberculous meningitis: clinical and computerized tomographic correlation. Dev Med Child Neurol. 1985;27(5):644-54.

119. Kosteljanetz M. CSF dynamics in patients with subarachnoid and/or intraventricular hemorrhage. J Neurosurg. 1984;60(5):940-6.

120. Hayashi M, Kobayashi H, Kawano H, Yamamoto S, Maeda T. Cerebral blood flow and ICP patterns in patients with communicating hydrocephalus after aneurysm rupture. J Neurosurg. 1984;61(1):30-6.

121. Brock M, Cervos-Navarro J, Holdorff B. Changes in intracranial pressure associated with delayed cerebral radionecrosis. Surg Neurol. 1984;22(1):8-16.

122. Kuchiwaki H, Kageyama N, Hirai N, Takada S, Inao S, Terashima M, et al A biological rhythm in a patient with normal pressure hydrocephaluscomparative studies in pre- and postoperative patients by a polygraphy. No To Shinkei. 1984;36(9):911-6.

123. Tamaki N, Kusunoki T, Kose S, Matsumoto S. Continuous intracranial pressure monitoring in normal pressure hydrocephalus-with special reference to clinical significance of B wave and prognostic criteria for CSF shunting. No To Shinkei. 1983;35(2):131-7. 
124. Auer LM, Sayama I. Intracranial pressure oscillations (B-waves) caused by oscillations in cerebrovascular volume. Acta Neurochir. 1983;68(1-2):93-100.

125. Terzano MG, Gatti PL, Manzoni GC, Formentini E, Mancia D. Is the EEG cyclic alternating pattern a true autonomous entity? Analytic study in a case of post-traumatic coma with good prognosis. Eur Neurol. 1982;21(5):324-34.

126. Gjerris F, Børgesen SE, Hoppe E, Boesen F, Nordenbo AM. The conductance to outflow of CSF in adults with high-pressure hydrocephalus. Acta Neurochir. 1982;64(1-2):59-67.

127. Tomei G, Gaini SM, Giovanelli M, Rampini P, Granata G, Villani R. Intracranial pressure in subarachnoid hemorrhage. Preliminary report in 36 cases. J Neurosurg Sci. 1981;25(2):57-66.

128. Kaye AH, Brownbill D. Postoperative intracranial pressure in patients operated on for cerebral aneurysms following subarachnoid hemorrhage. J Neurosurg. 1981;54(6):726-32.

129. Bilz D, Pásztor A, Paraicz E. Rhythmic correlations between regional intracranial blood volume and intracranial pressure within minutes during continuous simultaneous recording in infants. Psychiatr Neurol Med Psychol. 1981:33(8):483-90.

130. Munari C, Calbucci F, Casaroli D, Bonora M. Correlations between E.E.G. findings and intracranial pressure (ICP) during treatment of acute posttraumatic intracranial hypertension (preliminary report) (author's transl). Rev Electroencephalogr Neurophysiol Clin. 1980;10(4):319-30.
131. Guieu JD, Lapierre M, Blond S, Hurtevent JF, Jomin M, Pruvot P. Correlations between intracranial pressure variations and EEG changes in patients with cranial trauma (author's transl). Rev Electroencephalogr Neurophysiol Clin. 1979;9(2):194-201.

132. Wilkinson HA, Schuman N, Ruggiero J. Nonvolumetric methods of detecting impaired intracranial compliance or reactivity: pulse width and wave form analysis. J Neurosurg. 1979;50(6):758-67.

133. Liguori G, Fiordirosa M, Cicchiello LR, Ambrosio A. Long-term monitoring of intracranial pressure in hydrocephalic children not affected by cerebral tumor. J Neurosurg Sci. 1979;23(4):295-302.

134. Fuentes JM, Bouscarel C, Choucair Y, Roquefeuil B, Vlahovitch B, Blanchet P. Monitoring of intracranial pression in acute neurotrauma by extra-dural screw (author's transl). Anesth Analg. 1979;36(9-10):429-33.

135. Munari C, Calbucci F. Correlations between intracranial pressure (I.C.P.) and EEG changes during traumatic coma (author's transl). Rev Electroencephalogr Neurophysiol Clin. 1979;9(2):185-93.

136. Hayashi M, Marukawa S, Fujii H, Kitano T, Kobayashi H, Yamamoto S. Intracranial hypertension in patients with ruptured intracranial aneurysm. J Neurosurg. 1977;46(5):584-90.

\section{Publisher's Note}

Springer Nature remains neutral with regard to jurisdictional claims in published maps and institutional affiliations.
Ready to submit your research? Choose BMC and benefit from:

- fast, convenient online submission

- thorough peer review by experienced researchers in your field

- rapid publication on acceptance

- support for research data, including large and complex data types

- gold Open Access which fosters wider collaboration and increased citations

- maximum visibility for your research: over $100 \mathrm{M}$ website views per year

At BMC, research is always in progress.

Learn more biomedcentral.com/submissions 